This item was submitted to Loughborough's Research Repository by the author.

Items in Figshare are protected by copyright, with all rights reserved, unless otherwise indicated.

\title{
Reduced thymic output in elite athletes
}

PLEASE CITE THE PUBLISHED VERSION

http://dx.doi.org/10.1016/j.bbi.2014.01.004

\section{PUBLISHER}

(c) Elsevier

\section{VERSION}

AM (Accepted Manuscript)

\section{PUBLISHER STATEMENT}

This work is made available according to the conditions of the Creative Commons Attribution-NonCommercialNoDerivatives 4.0 International (CC BY-NC-ND 4.0) licence. Full details of this licence are available at: https://creativecommons.org/licenses/by-nc-nd/4.0/

\section{LICENCE}

CC BY-NC-ND 4.0

\section{REPOSITORY RECORD}

Prieto-Hinojosa, Adria, Andrea Knight, Claude Compton, Michael Gleeson, and Paul J. Travers. 2019. "Reduced Thymic Output in Elite Athletes". figshare. https://hdl.handle.net/2134/15713. 


\section{Accepted Manuscript}

Reduced thymic output in elite athletes

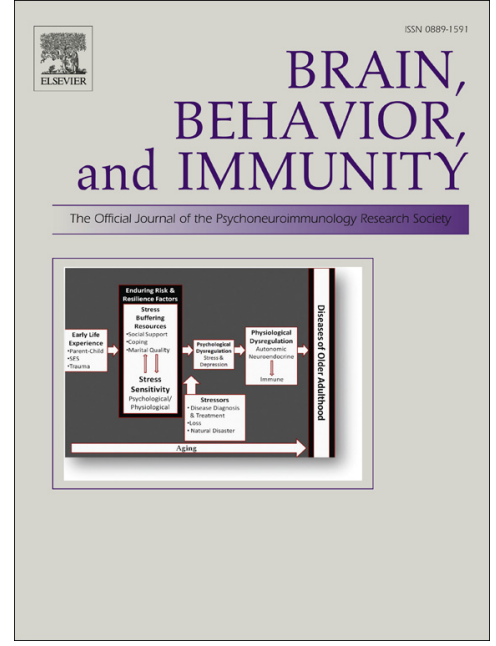

Adria Prieto-Hinojosa, Andrea Knight, Claude Compton, Michael Gleeson, Paul J Travers

PII:

S0889-1591(14)00005-1

DOI:

http://dx.doi.org/10.1016/j.bbi.2014.01.004

Reference:

YBRBI 2291

To appear in:

Brain, Behavior, and Immunity

Received Date: $\quad 31$ July 2013

Revised Date: $\quad 18$ December 2013

Accepted Date: 7 January 2014

Please cite this article as: Prieto-Hinojosa, A., Knight, A., Compton, C., Gleeson, M., Travers, P.J., Reduced thymic output in elite athletes, Brain, Behavior, and Immunity (2014), doi: http://dx.doi.org/10.1016/j.bbi.2014.01.004

This is a PDF file of an unedited manuscript that has been accepted for publication. As a service to our customers we are providing this early version of the manuscript. The manuscript will undergo copyediting, typesetting, and review of the resulting proof before it is published in its final form. Please note that during the production process errors may be discovered which could affect the content, and all legal disclaimers that apply to the journal pertain. 


\section{Reduced thymic output in elite athletes}

Adria Prieto-Hinojosa ${ }^{1}$, Andrea Knight ${ }^{1}$, Claude Compton ${ }^{2}$, Michael Gleeson ${ }^{3}$ and Paul J Travers ${ }^{1,4}$

${ }^{1}$ Anthony Nolan Research Institute, The Royal Free Hospital, London ${ }^{2}$ School of Life Sciences University of Edinburgh

${ }^{3}$ School of Sport and Exercise Science, Loughborough University

${ }^{4}$ MRC Centre for Regenerative Medicine, Edinburgh Royal Infirmary

Running head: Thymic Output in Elite Athletes

Corresponding Author

Paul J Travers

MRC Centre for Regenerative Medicine,

SCRM Building

Edinburgh Royal Infirmary,

Edinburgh EH16 4UU

United Kingdom

Phone: +44(0)1316519548

Fax: :+44 (0)1316519501

Email: paul.travers@ed.ac.uk

Keywords: Exercise Immunology; Thymus; Naïve T cell 


\section{Abstract}

Athletes undergoing intensive training schedules have chronic exposure to stress-induced hormones such as cortisol that can depress immune function. We compared the circulating levels of T cell receptor excision circles (TREC), a marker of recent thymic emigrants, as well as the levels of naïve and memory subsets in a group of elite endurance athletes and in controls. The athletes showed a reduction in absolute numbers of naïve T cells, particularly in CD4 T cells. In contrast, memory cells were increased. TREC levels in the athletes were significantly reduced compared to age-matched controls. Such changes resemble premature ageing of the T cell component of the immune system. Since thymic production of $\mathrm{T}$ cells naturally decline with age, these results raise the concern that prolonging high intensity exercise into the $4^{\text {th }}$ decade of life may have deleterious consequences for athletes' health. 


\section{Introduction}

The activity of the thymus, an essential organ for the production of T cells, declines with age (Grey et al, 1975) (Fallen et al., 2003b; Jamieson et al., 1999; Kurashima et al., 1995; McFarland et al., 2000). Consequent upon this decline is a change in the distribution of cells in the peripheral circulation, with naïve $\mathrm{T}$ cells decreasing with age while memory and effector cells increase. Such changes are more extreme either when the thymus has been removed early in life(Gress and Deeks, 2009), or when there is profound lymphopenia requiring reconstitution of the peripheral lymphoid compartment, as after haematopoietic stem cell transplantation(Fallen et al., 2003b). In this latter case it is clear that in patients over 30 years of age the contribution of the thymus to repopulation of the peripheral T cell pool is significantly impaired and distortions in the distribution of different $\mathrm{T}$ cell subsets can be maintained for some years after the transplant itself(Fallen et al., 2003a). Such changes in the distribution of T cell subsets may not be reflected in the peripheral T cell numbers, since a constant level of T cells may be maintained through expansions and contractions of different subsets. Measurement of thymus activity is thus essential to understand the relative contributions of the thymus versus peripheral expansion in maintaining T cell numbers. Signal-joint T cell receptor excision circles (SjTREC) are a marker of the commitment of T cells to the $\alpha \beta$ lineage during intrathymic development (Koup et al., 1998); thus the concentration of sjTREC in peripheral $\mathrm{T}$ cells can provide an indication of the activity of the thymus.

Signal-joint T cell receptor excision circles (sjTREC) are a marker of the commitment of T cells to the $\alpha \beta$ lineage during intrathymic development (Koup et al., 1998); thus the concentration of sjTREC in peripheral T cells can provide an indication of the activity of the thymus. We therefore compared $\mathrm{T}$ cell subset distribution and sjTREC levels between an elite endurance athlete cohort and a group of age matched healthy controls in order to measure the impact of chronic exposure to stress in otherwise young, healthy individuals. 


\section{Materials and Methods}

\section{Donor cohorts}

The athlete cohort comprised a group of 19 national standard triathletes training at Loughborough University; each individual trained for a minimum of 6 days a week and for a minimum of 1.5 hours per session, swimming, running and cycling between 280-400 km per week plus 8 hours per week of weight, interval or skills training. The age range in this cohort was 18-36, with a median age of 20.7 yrs.

The age-matched control cohort comprised students from the Universities of Bath, Edinburgh and University College, London selected only on the basis of an age between 18-36 yrs at the time of sampling, with a median age of 21 . A second control group, drawn largely from volunteer co-workers, had an age range from 30-55. While healthy, the control donors reported no systematic endurance training programs.

\section{Blood samples}

20-40 ml samples of peripheral blood were taken by sterile venepuncture after informed consent from each donor under approval by the relevant institutional ethics committees. Samples were taken from the athletes on a rest day, at least 18 hours after the last exercise session.

Absolute cell counts were obtained from fresh unseparated blood using BD TruCount tubes and the BD MultiTest CD3-FITC/CD8-PE/CD45-PerCP/CD4-APC reagents; data were collected and analysed using BD Multiset software on a BD FACScan flow cytometer (all BD Biosciences, San Jose,CA).

Peripheral blood mononuclear cells were prepared by density gradient centrifugation with a lower layer of Lympholyte (Cedarlane Laboratories Limited, USA) on which PBMC float. These were recovered, washed twice in 
RPMI1640, resuspended in $10 \mathrm{ml}$ RPMI 1640 and the cell yield counted using an haemocytometer (Neubaur Chamber, Weber).

\section{Flow Cytometry}

Cells stained with fluorochrome-conjugated antibodies were visualised using flow cytometry using FACSCalibur flow cytometer and CellQuest software (BD Bioscience, San Jose, California, USA). FloJo (Tree Star Inc, Ashland, Oregon, USA) was used for analysis of stained subsets. The antibodies used for analysis were CD3-APC (clone HIT3a), CD4-PerCP (clone SK3), CD8-PerCP (clone SK1), CD27FITC (clone M-T271), CD45RA-FITC (clone L48), and CD45R0-PE (clone UCHL1) (all BD Bioscience, San Jose, California, USA).

\section{Selection of CD4 and CD8 subsets from PBMC's}

Isolation of CD4+ and CD8+ T-cells was carried out using EasySep positive magnetic separation (Stem Cell Technologies, Vancouver, Canada) according to the manufacturer's instructions and positive selection MS+/RS+ columns with a maximum capacity of $1 \times 10^{7}$ labelled cells.

\section{Quantitative PCR measurement of TREC numbers}

Real-Time PCR was carried out using an ABI/PRISM 7500 sequence detection system and SDS software (Applied Biosystems, Foster City, CA) to quantify sjTREC in cell lysates from donor and athlete samples. The 5' primer, 3' primer and probe sequences were CACATCCCTTTCAACCATGCT, GCCAGCTGCAGGGTTTAGG and FAM-ACACCTCTGGTTTTTGTAAAGGTGCCCACTTAMRA respectively.

PCR reactions were set up in 96-well optical reaction plates (Applied Biosystems, Foster City, CA, USA); sealed using Optical Adhesive Covers (Applied Biosystems, Foster City, CA, USA) and transferred to the ABI/PRISM 7500 Sequence Detector for analysis. 
To quantify sjTREC a series of standard dilutions of plasmid containing the signal-joint breakpoint was created following a standard protocol (Douek et al., 2000). Each sample was run in triplicate along with the dilution series of TREC plasmid and 'no template' control (NTC).

The PCR was carried out in $25 \mu \mathrm{l}$ reactions containing $5 \mu \mathrm{l}$ of cell lysates or $5 \mu \mathrm{l}$ of the standard, and a final concentration of $1 \mathrm{U}$ Platinum Taq (Invitrogen Ltd., USA), $3.5 \mathrm{mM} \mathrm{MgCl}_{2}, 0.25 \mathrm{mM}$ dNTPs, $12.5 \mathrm{mM}$ of each primer, $3.75 \mathrm{mM}$ fluorescent labelled probe, $2.5 \mathrm{ml}$ Platinum buffer and $0.5 \mathrm{ml}$ of ROX reference dye. One cycle of denaturation ( $95^{\circ} \mathrm{C}$ for 5 minutes) was performed, followed by 40 cycles of amplification ( $95^{\circ} \mathrm{C}$ for $30 \mathrm{sec}, 60^{\circ} \mathrm{C}$ for 1 minute).

\section{$\underline{\text { Statistical Analysis }}$}

Statistical analyses were carried out using Prism (Graphpad Software Inc, La Jolla, CA, USA). Comparison between the athlete and individual control groups was made using the non-parametric Mann-Whitney test. In addition, comparison between all groups was made using the Kruskal-Wallis test and adjusted with Dunn's multiple comparison test. A p value of 0.05 was taken as the threshold for significance.

\section{$\underline{\text { Results }}$}

\section{Peripheral CD3 $\mathrm{T}$ cell counts in athletes are the same as those in controls yet thymic output is significantly reduced.}

As exercise has been shown to induce apoptosis of thymocytes(Concordet and Ferry, 1993), we sought to evaluate the impact of chronic exercise stress on the output of new T cells. Previous studies have shown that exercise causes transient changes in lymphocyte numbers - a lymphocytosis followed by a lymphopenia that resolves over 3-12 hours. To determine whether this might influence the results obtained, we measured the levels of CD3+ cells ( $T$ cells) in the peripheral circulation of the athletes on a rest day (at least 16 hours after exercise) and compared the values obtained to those of normal, healthy, agematched controls, as well as to an older healthy control group. None of the 
values obtained - means of $1456 \pm 420 \mathrm{CD} 3+$ cells per ul of blood for the athletes versus $1411 \pm 467$ for the matched controls and $1421 \pm 742$ for the older controls - are significantly different from any other.

To ask whether there was a differential contribution of thymic output to the peripheral T cell populations, we compared the sjTREC concentration in the peripheral blood of the athletes with that of age-matched controls, as shown in Figure 1. Although there is no significant difference in the number of T cells in the peripheral circulation comparing athletes with age-matched controls, we observed a striking and highly significant $(\mathrm{p}<0.0001)$ reduction in the sjTREC number in the athletes. The median sjTREC count in the control cohort is 3.20 $\mathrm{x} 10^{4}$ per $\mathrm{ml}$ versus $0.13 \times 10^{4}$ per $\mathrm{ml}$ in the athlete cohort. In normal, healthy individuals, values as low as those seen in the athletes are not generally observed until at least 60 years of age(Jamieson et al., 1999; McFarland et al., 2000).

\section{Different rates of thymus-dependent replacement of CD4 and CD8 T cells in the athletes}

To ask whether the decline in sjTREC observed affected all T cell subsets equally, the contribution of the thymus was assessed by quantitating sjTRECs within purified CD4 and CD8 T cell populations, as shown in Figure 2. A different distribution of sjTREC was observed between the two subsets, with significantly lower counts being detected in the athletes' CD4 T cells compared to those within the CD8 T cells.

\section{CD4 and CD8 $\mathrm{T}$ cell subsets differ in athletes from controls}

As shown in Figure 3, which shows the number of cells of each subset per $\mu$ l of whole blood, the naïve (CD45RA $\left.{ }^{+} \mathrm{CD} 27^{+}\right) \mathrm{CD}^{+} \mathrm{T}$ cell subset is significantly depleted in the athletes versus age-matched controls ( $\mathrm{p}<0.0001$; upper left). There is a lesser but still significant depletion relative to the cohort of older individuals ( $\mathrm{p}=0.049)$. The naïve CD8 subset is also depleted in the athlete 
cohort relative to the age-matched controls ( $\mathrm{p}=0.0034$; upper right), and to the older control cohort $(\mathrm{p}=0.019)$.

Conversely, the CD4 and CD8 memory subsets, defined as being CD45RO+, are expanded in the athlete cohort compared to the age-matched normal controls. The CD $4^{+}$memory subset (Figure 3; lower left) shows a significant increase compared to the age-matched controls $(\mathrm{p}=0.0008)$ but not to the older control cohort ( $\mathrm{p}=0.0868)$ while the $\mathrm{CD}^{+}$memory subset (Figure 3 ; lower right) is significantly expanded in the athletes versus both age-matched $(\mathrm{p}<0.0001)$ and older $(\mathrm{p}<0.0001)$ control cohorts.

There were no significant differences between the age matched and older control cohorts for any of the $\mathrm{T}$ cell subsets.

\section{Discussion}

Our observations in the cohort of young athletes compared to age-matched controls show clearly that the athletes have a depleted naïve T cell compartment compared to the controls. In particular the contribution of the thymus to maintaining the peripheral naïve T cell pool appears to be significantly diminished, since the numbers of sjTREC molecules detected in the athletes is reduced compared to the age-matched controls. Moreover, it is also reduced compared to the older controls.

The first question to ask is whether these results reflect a loss of the T cells themselves, or simply a sequestration of the cells such that they are not detectable in the peripheral circulation. This has an important consequence in that were the cells to be sequestered as a consequence of intensive training, then there could be a rapid return to normal levels on cessation of training, whereas is the cells are indeed lost, the only route to the restoration of normal levels would by through new production of T cells via the thymus. As thymic activity declines with age, recovery by this route would lead to a delay in older athletes. Were the naïve cells to be sequestered, there should be no effect on the numbers of memory $\mathrm{T}$ cells, yet we measure a significant increase in the absolute 
numbers of memory $\mathrm{T}$ cells which compensates for the observed reduction in naïve T cells. The most likely interpretation of our data therefore is that there is a loss of naïve T cells. The decrease in sjTREC and the increase in memory T cells suggest that this loss is the result of a chronic process where naïve cells, driven into the memory phenotype by exposure to antigen, are not being replaced by the production of new naïve $\mathrm{T}$ cells from the thymus.

The pattern of reduced naïve T cell numbers, reduced sjTREC and compensating expansions in memory and effector $\mathrm{T}$ cells resembles that seen in ageing where thymic function is reduced (Czesnikiewicz-Guzik et al., 2008; Kurashima et al., 1995). Likewise after haematopoietic stem cell transplant, older individuals with reduced thymic output show a pattern of reconstitution with decreased numbers of naïve $\mathrm{T}$ cells and a skewing of $\mathrm{T}$ cell subsets towards late memory and effector T cells (Fallen et al., 2003b; Komanduri et al., 2007).

We have asked to what extent our results could be a consequence not of some difference in the production of new naïve $\mathrm{T}$ cells in the athletes, but reflect the impact of large expansions of memory and effector cell populations driven by a differential exposure to, or susceptibility to, infections. However, we do not believe that this can account for the observations we have made. Firstly, the total $\mathrm{T}$ cell counts in the athletes and controls do not differ, so we have no evidence for any lymphocytosis that might dilute the naïve T cell pool. Second, while it is the case that measurements of sjTREC per fixed number of CD3+ or CD4+or CD8+ cells would be decreased as a consequence of expansion of activated T cells, we initially measured sjTREC per ml of blood, which would be unaffected by changes in the numbers of memory and effector cells present in the same sample. Finally, we also measured absolute numbers of naïve and memory cells in the athletes and controls, and here again the measurement of the naïve $\mathrm{T}$ cell pool is unaffected by expansions of the memory pool. 
As mentioned above, differential expansion or contraction of different T cell subsets can affect TREC count for a given cell number, which can thus be influenced by homeostatic drive or by prior history of infection. These factors could influence the interpretation of the thymic input into the separated CD4 and CD8 T cell subsets. Previous measurements of sjTREC levels in separated CD4 and CD8 cells (Schonland, 2003; Hankey, 2007) show that sjTREC levels in the CD4 subset in adults are approximately $60 \%$ of those seen in CD8 T cells. This is in contrast to levels in umbilical cord blood (Schonland, 2003; Hankey, 2007) and in young ( $<30$ years of age) patients reconstituting after haematopoietic stem cell transplant (Fallen et al., 2003b), where the sjTREC levels in the CD4 and CD8 subsets are equivalent. Both of these latter observations are situations where the naïve $\mathrm{T}$ cell levels directly reflect thymic output. Thus any disparity in sjTREC levels between the CD4 and CD8 subsets in the athletes is likely to reflect differential cell loss and differences in both homeostatic and antigen driven proliferation. A greater reliance on peripheral T cell expansion in the athletes rather than new thymic production of T cells results in a more profound dilution effect in the athletes; the median sjTREC level (104.07) in athlete CD4 T cells represents only 15\% respectively of the median (683.41) level present in the CD8 T cells. compared to the ratio of approximately 0.6 seen in normal individuals(Schonland, 2003; Hankey, 2007).

The overall picture of the peripheral T cell compartment in the athletes, whether derived from total sjTREC, the distribution of sjTREC in the CD4 and CD8 subsets, or the distribution of naïve and memory cells, particularly within the CD4 subset, is consistent with that of thymic insufficiency. The underlying cause of this insufficiency is unclear and we would wish to investigate this further. Corticosteroids and the stress response have been shown to contribute to thymic involution and in human and animal studies, exercise and environmental stress have been associated with a decrease in thymus weight(Kioukia-Fougia, 2002; Selye, 1936), while exercise has been shown to induce apoptosis in both thymocytes(Concordet and Ferry, 1993) and peripheral blood 
lymphocytes(Mooren et al., 2002). Glucocorticoids, notably cortisol, appear to mediate this effect in both environmental(Tarcic et al., 1998) and exercise stress(Kruger et al., 2011) models and further studies, including direct measurements of cortisol levels, are needed to determine glucocorticoids are indeed the basis for the reduced thymic function we observe in the athlete cohort.

Given the reduced levels of naïve T cells and the reduced thymic output we observe, the question arises: whether and how the athletes will recover from this state? It is clear from studies of immune reconstitution after intensive chemotherapy or stem cell transplantation for haematological malignancies that the pattern of $\mathrm{T}$ cell recovery is dependent on thymus function. From our previous work, we defined a threshold age of 30 , with patients below 30 years of age showing a pattern of immune reconstitution characterised by increasing naïve T cells and increasing sjTREC numbers, while older patients show prolonged periods of relative immune deficiency characterised by reduced numbers of naïve T cells and expanded memory and effector cells. In the extreme case, athletes prolonging their careers into their 30s (or beyond) may run the risk of passing from an exercise-induced thymic insufficiency to an agedependent one, creating a permanent distortion, in effect a premature ageing, in their peripheral $\mathrm{T}$ cell repertoire that leaves them at reduced capacity to respond to new infectious challenges. The changes in the immune system in the elderly leave them at increased risk of infectious morbidity and mortality (Pawelec et al., $2010 \mathrm{~b}$ ) and may play a role in the increased risk of cancer seen in the elderly (Pawelec et al., 2010a). Moreover, since the thymus also produces regulatory CD4 T cells that act to suppress autoimmune responses, long term depletion of the CD4 subset is likely to lead to increased autoimmunity, such as is also seen in ageing (Grolleau-Julius et al., 2009). Our data are important in that they suggest the possibility that athletes are at risk of long term immune dysfunction. The extent to which these effects on the immune system persist on cessation (or substantial reduction) of exercise training is not known, and will need to be established if the long term health of athletes is to be protected. 


\section{Acknowledgements}

We would like to thank Rose Zamoyska for critical discussion of the manuscript and Dr Cornelia Weyand for communicating data on TREC counts in normal CD4 and CD8 subsets. We acknowledge the support of both the EU FP6 project "Allostem", and the University of Edinburgh in funding this work. 


\section{References}

Concordet, J.P., Ferry, A., 1993. Physiological programmed cell death in thymocytes is induced by physical stress (exercise). Am. J. Physiol. 265, C626-9.

Czesnikiewicz-Guzik, M., Lee, W.-W., Cui, D., Hiruma, Y., Lamar, D.L., Yang, Z.-Z., Ouslander, J.G., Weyand, C.M., Goronzy, J.J., 2008. T cell subset-specific susceptibility to aging. Clinical Immunology 127, 107-118.

Douek, D.C., Vescio, R.A., Betts, M.R., Brenchley, J.M., Hill, B.J., Zhang, L., Berenson, J.R., Collins, R.H., Koup, R.A., 2000. Assessment of thymic output in adults after haematopoietic stemcell transplantation and prediction of T-cell reconstitution. The Lancet 355, 1875-1881.

Fallen, P.R., Duarte, R.F., McGreavey, L., Potter, M., Ethell, M., Prentice, H.G., Madrigal, J.A., Travers, P.J., 2003a. Identification of non-naïve CD4+CD45RA+ $\mathrm{T}$ cell subsets in adult allogeneic haematopoietic cell transplant recipients. Bone Marrow Transplant 32, 609-616.

Fallen, P.R., McGreavey, L., Madrigal, J.A., Potter, M., Ethell, M., Prentice, H.G., Guimarães, A., Travers, P.J., 2003b. Factors affecting reconstitution of the T cell compartment in allogeneic haematopoietic cell transplant recipients. Bone Marrow Transplant 32, 1001-1014.

Gress, R.E., Deeks, S.G., 2009. Reduced thymus activity and infection prematurely age the immune system. J. Clin. Invest. 119, 2884-2887.

Grolleau-Julius, A., Ray, D., Yung, R.L., 2009. The Role of Epigenetics in Aging and Autoimmunity. Clinic Rev Allerg Immunol 39, 42-50.

Hankey, K. Thymic Contribution To The Generation and Maintenance of the Peripheral T-cell Pool In Healthy and Lympho-depleted Subjects. PhD Thesis, University of Maryland, Baltimore, 2007.

Gray, E.S, Beck, J.S., Simpson, J. G., 1975. Age involution in the normal human adult thymus. Clin Exp Immunol 19, 261.

Jamieson, B.D., Douek, D.C., Killian, S., Hultin, L.E., Scripture-Adams, D.D., Giorgi, J.V., Marelli, D., Koup, R.A., Zack, J.A., 1999. Generation of functional thymocytes in the human adult. Immunity 10, 569-575.

Kioukia-Fougia, N., 2002. The effects of stress exposure on the hypothalamicpituitary-adrenal axis, thymus, thyroid hormones and glucose levels. Progress in Neuro-Psychopharmacology and Biological Psychiatry 26, 823830.

Komanduri, K.V., St John, L.S., de Lima, M., McMannis, J., Rosinski, S., McNiece, I., Bryan, S.G., Kaur, I., Martin, S., Wieder, E.D., Worth, L., Cooper, L.J.N., Petropoulos, D., Molldrem, J.J., Champlin, R.E., Shpall, E.J., 2007. Delayed immune reconstitution after cord blood transplantation is characterized by impaired thymopoiesis and late memory T-cell skewing. Blood 110, 45434551.

Koup, R.A., Douek, D.C., McFarland, R.D., Keiser, P.H., Gage, E.A., Massey, J.M., Haynes, B.F., Polis, M.A., Haase, A.T., Feinberg, M.B., Sullivan, J.L., Jamieson, B.D., Zack, J.A., Picker, L.J., 1998. Nature 396, 690-695.

Kruger, K., Agnischock, S., Lechtermann, A., Tiwari, S., Mishra, M., Pilat, C., Wagner, A., Tweddell, C., Gramlich, I., Mooren, F.C., 2011. Intensive resistance exercise induces lymphocyte apoptosis via cortisol and glucocorticoid receptor-dependent pathways. Journal of Applied Physiology 110, 12261232. 
Kurashima, C., Utsuyama, M., Kasai, M., Ishijima, S.A., Konno, A., Hirokawa, K., 1995. The role of thymus in the aging of Th cell subpopulations and ageassociated alteration of cytokine production by these cells. Int. Immunol. 7 , 97-104.

McFarland, R.D.R., Douek, D.C.D., Koup, R.A.R., Picker, L.J.L., 2000. Identification of a human recent thymic emigrant phenotype. Proc Natl Acad Sci U S A 97, 4215-4220.

Mooren, F.C., Blöming, D., Lechtermann, A., Lerch, M.M., Völker, K., 2002. Lymphocyte apoptosis after exhaustive and moderate exercise. J. Appl. Physiol. 93, 147-153.

Pawelec, G., Derhovanessian, E., Larbi, A., 2010a. Immunosenescence and cancer. Crit. Rev. Oncol. Hematol. 75, 165-172.

Pawelec, G., Larbi, A., Derhovanessian, E., 2010b. Senescence of the human immune system. Journal of Comparative Pathology 142 Suppl 1, S39-44.

Schonland, S.O., 2003. Homeostatic control of T-cell generation in neonates. Blood 102, 1428-1434.

Selye, H., 1936. A syndrome produced by diverse nocuous agents. Nature 138, 32-32.

Tarcic, N., Ovadia, H., Weiss, D.W., Weidenfeld, J., 1998. Restraint stress-induced thymic involution and cell apoptosis are dependent on endogenous glucocorticoids. J Neuroimmunol 82, 40-46. 
Figure Legends

Figure 1 Thymic function is significantly diminished in athletes versus agematched controls

qPCR measurements of sjTREC numbers were obtained for the athletes and agematched controls. Symbols represent individual values (means of triplicates) while the horizontal bars show the mean values obtained (control: $3.4 \times 10^{4} \pm 0.65 \times 10^{4}$; athlete: $0.5 \times 10^{4} \pm 0.8 \times 10^{4}$ ). The two cohorts differ significantly $(\mathrm{p}<0.0001)$.

Figure 2 sjTREC counts are significantly lower in athlete CD4 versus CD8 T cell subsets

Quantitative PCR of sjTREC was carried out on lysates from a known number of purified CD4 and CD8 T cells and the results displayed as sjTREC per 150,000 cells. The symbols show individual values while the horizontal bars show the mean values obtained - $292 \pm 395$ in the CD4 subset versus $2090 \pm 2706$ in the CD8 subset. The populations are significantly different $(p=0.0194)$.

Figure 3 Athletes have fewer naïve T cell counts and more memory T cells compared to controls

Naïve and memory CD4 and CD8 T cells were identified on the basis of cell surface marker phenotypes, with naïve cells being CD3+CD27+CD45R0- and memory cells being CD3+CD45RO+ in addition to being CD4+ or CD8+ respectively. Cell counts were obtained from the percentage of each subset within CD3+ cells and the absolute count of CD3+ cells obtained using TruCount tubes and the BD TriTest reagent. Symbols show values obtained for each subset from the athletes, age matched controls and the older control cohort, while the horizontal bars show the mean values for each cohort. The individual means and standard deviations obtained are: 
Naïve CD4 T cells: athlete $252 \pm 212$; age-matched control $524 \pm 216$; older control $462 \pm 262$

Naïve CD8 T cells: athlete $158 \pm 125$; age-matched control 253 \pm 124 ; older control $232 \pm 125$

Memory CD4 T cells: athlete 489 \pm 161 ; age-matched control 308 \pm 94 ; older control $427 \pm 341$

Memory CD8 T cells: athlete 341 \pm 153 ; age-matched control 126 \pm 73 ; older control $144 \pm 104$ 
Figure 1

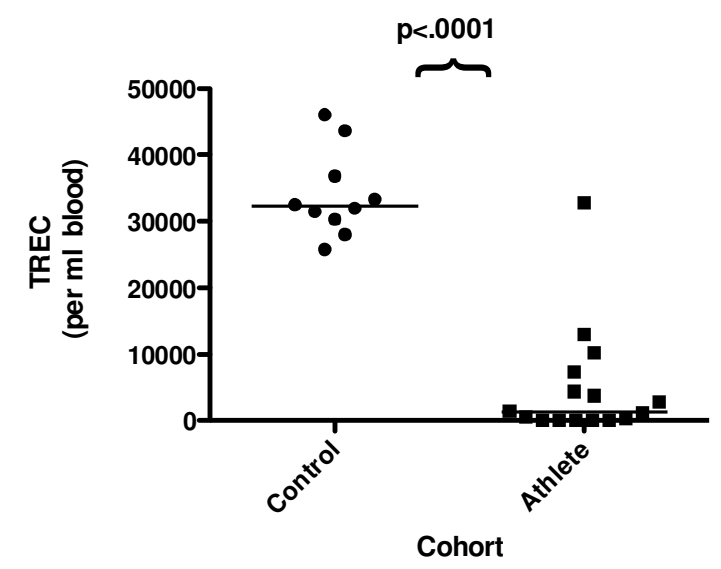

Figure 2

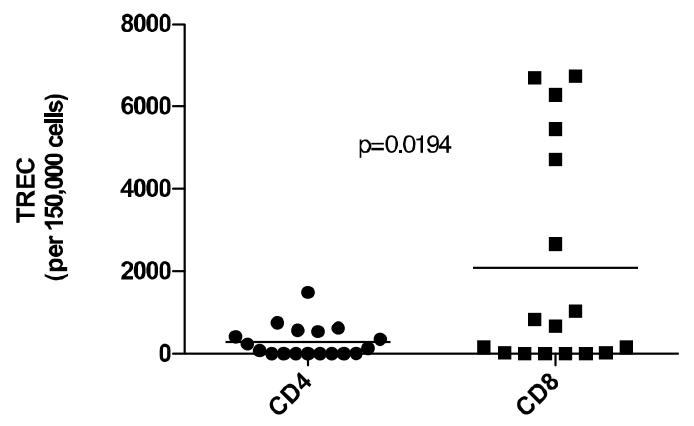


Figure 3

Naive CD4 T cells

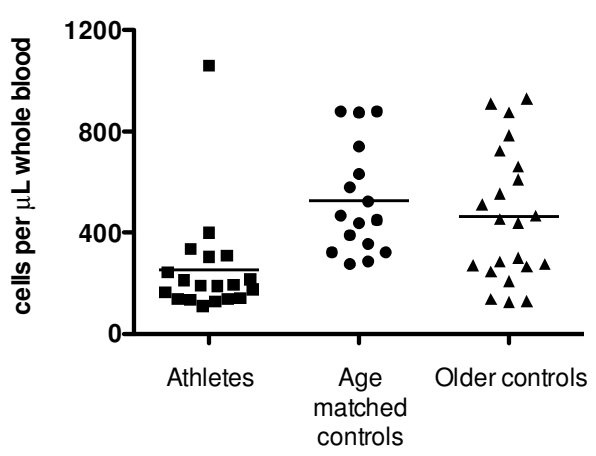

Memory CD4 T cells

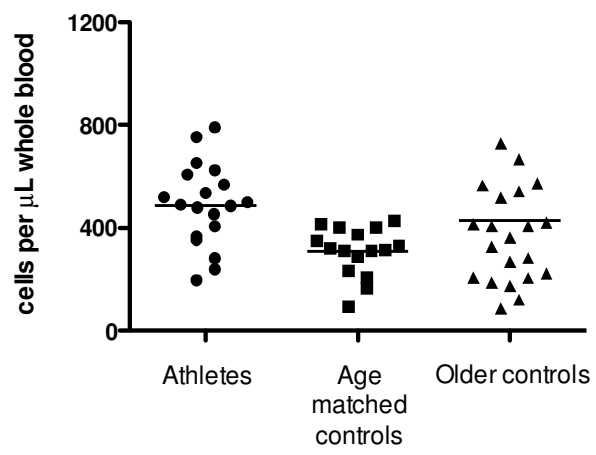

Naive CD8 T cells

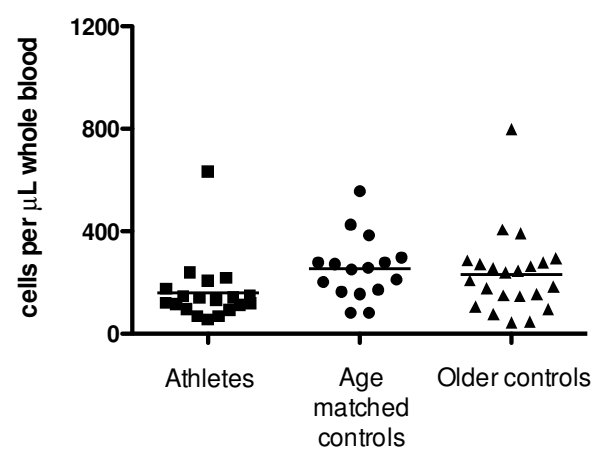

Memory CD8 T cells

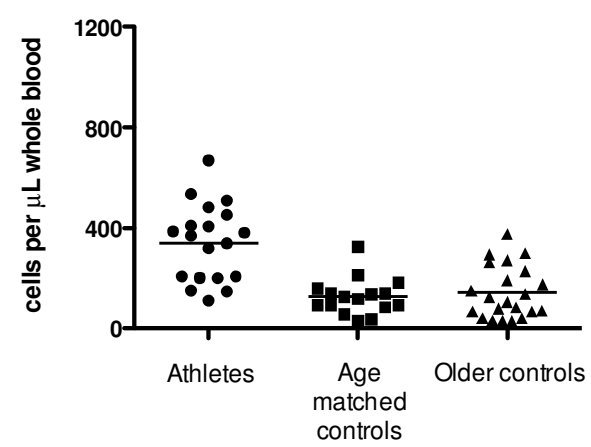


Elite athletes show low numbers of naïve T cells and evidence of decreased thymic function, a finding which has potential long term implications for their health. 